\title{
UNE TECHNIQUE SIMPLE POUR L'ANALYSE GRANULOMETRIQUE DE LE TOURBE EN MILIEU AQUEUX
}

Le tamisage de la tourbe comme mode opératoire dans l'analyse granulométrique ou pour la détermination du contenu en fibre $(>0.15 \mathrm{~mm})$ est une opération qui est de plus en plus fréquente en laboratoire. Pour les fins ci-devant mentionnées, le tamisage est fait en milieu aqueux afin d'éviter l'influence du séchage sur la nature de la tourbe; de plus, les particules organiques, étant très légères, ont besoin d'un certain effet d'entraînement.

Sans doute, existe-il des techniques pour tamiser la tourbe en milieu aqueux (wet sieving), comme celle décrite par Boelter (1969). Cependant, en pareil cas, il est toujours difficile de régulariser ou d'uniformiser le volume et la pression du courant d'eau devant laver les fibres et entraîner les particules à travers les tamis. Le tamisage peut s'opérer aussi par la méthode de l'immersion complète des tamis (Bourget et Kemp 1957). Mais cette méthode ne peut assurer une bonne ségrégation des particules à cause de la constante remontée des éléments plus fins dans les tamis supérieurs.

Le dispositif qui est montré dans la figure 1 permet un bon tamisage de la tourbe; de plus, il a l'avantage d'être simple de conception et d'opération. L'ensemble comprend un cylindre de $46 \mathrm{~cm}$ de haut et d'un diamètre intérieur de $14.5 \mathrm{~cm}$; une ouverture au bas permet soit l'entrée de l'air (A) ou la sortie de l'eau (B). Un tuyau de caoutchouc (C) de $1.5 \mathrm{~cm}$ est placé au fond, sur le pourtour du cylindre; il sert à libérer le fond du dernier tamis en même temps qu'à amortir le mouvement dansant des tamis. Les tamis $(13.5 \mathrm{~cm})$ auxquels on a ajouté un mince tube (D) de tygon $(2 \mathrm{~mm})$ pour assurer un joint hermétique entre eux sont assemblés et retenus ensemble au moyen d'un élastique résistant. Un entonnoir (E) ferme l'ouverture du cylindre; il permet l'introduction de l'échantillon en même temps qu'il prévient les pertes d'eau

Can. J. Soil Sci. 56: 119-120 (May 1976) qui pourrait être projetée au dehors durant l'opération.

\section{Mode opératoire}

L'échantillon de tourbe $(25 \mathrm{~g}$ dans $300 \mathrm{ml}$ d'eau), après avoir été brassé pendant $16 \mathrm{~h}$, est d'abord passé au plus fin des tamis et lavé dans un bain d'eau pour receuillir la fraction qui est plus petite que 200-mesh. Ensuite, après la mise en place des tamis dans le cylindre, l'échantillon est placé sur le tamis du haut (10-mesh) et le cylindre est rempli d'eau (E) jusqu'au niveau du tamis supérieur de façon à ce que l'échantillon soit légèrement submergé. C'est alors qu'on

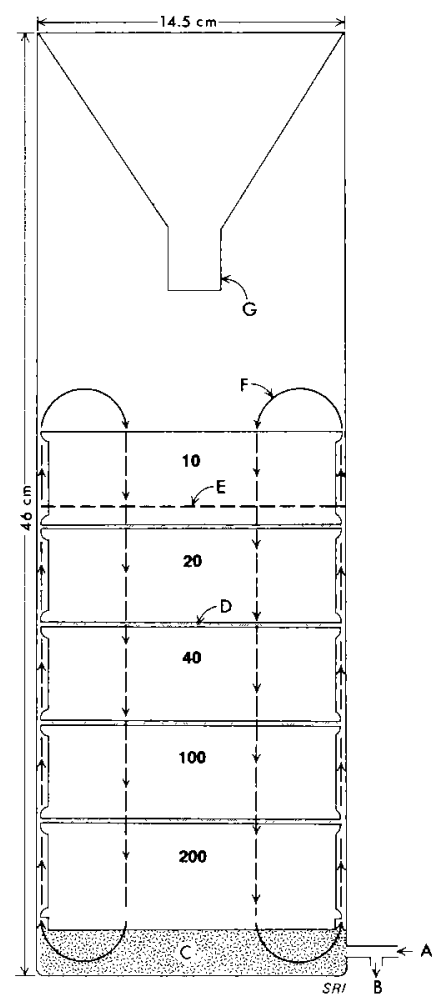

Fig. 1. Assemblage des tamis dans le cylindre devant servir à l'analyse granulométrique de la tourbe. 


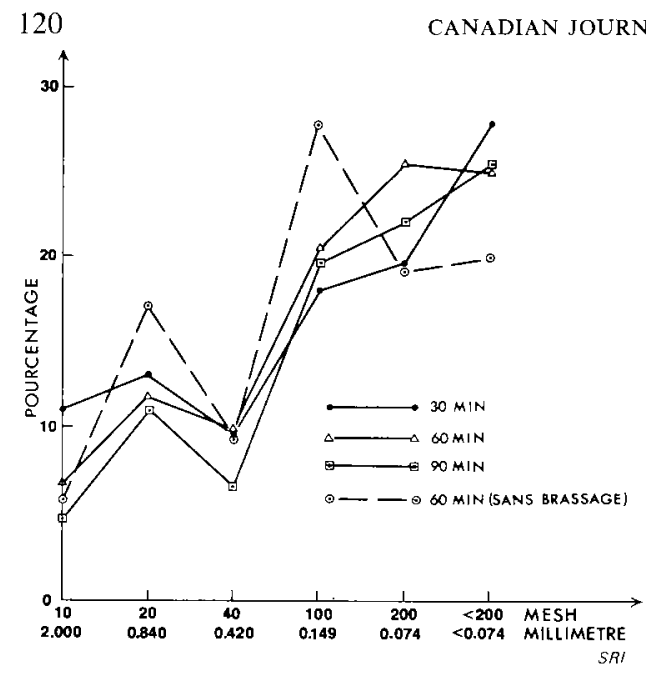

Fig. 2. Distribution des fractions granulométriques d'une tourbe de laîches (Ormstown, Qué.) ayant été soumise à trois différents temps de tamisage.

introduit de l'air par l'orifice du bas (A); une turbulence est créée sous l'effet des bulles d'air qui viennent se briser au haut des tamis, entraînant une certaine quantité d'eau qui retombe sur le tamis. Ainsi, l'eau lave les fibres et entraine les particules vers le bas. Cet entraînement est favorisé par l'action des bulles d'air qui font danser les tamis sur le tuyau placé au bas du cylindre.

Après une heure d'activité dans le cylindre, la distribution des particules sur les différents tamis a pris une allure définitive et l'opération peut être considérée comme terminée. Il est entendu que quelques précautions sont nécessaires si l'on veut être en mesure de reproduire les résultats. C'est ainsi que pour obtenir une bonne dispersion de la tourbe, il faut la cisailler en petits cubes et la soumettre à une période de brassage $(16-20 \mathrm{~h})$. Une tourbe de laîches (Ormstown, Qué.), de nature mésique a été soumise à trois différentes périodes de tamisage $(30,60$ et $90 \mathrm{~min}$ ). Comme l'indique la figure 2 qui représente la situation où l'on a obtenu le plus de différence, la période de tamisage n'est pas très critique; par contre, la distribution des particules est changée si la période de brassage est omise.

BOELTER, D. H. 1969. Physical properties of peats as related to degree of decomposition. Soil Sci. Soc. Amer. Proc. 33: 606-609.

BOURGET, S. J. et KEMP, J. G. 1957. Wet sieving apparatus for stability analysis of soil aggregates. Can. J. Soil Sci. 37: 60.

\section{H. DINEL et M. LEVESQUE}

Soil Research Institute, Agriculture Canada, Ottawa, Ontario K1A 0C6. Contribution no. 542, reçu le I mai 1975, acceptée le 8 juillet 1975. 